九州大学学術情報リポジトリ

Kyushu University Institutional Repository

\title{
A Hardy's Uncertainty Principle Lemma in Weak Commutation Relations of Heisenberg-Lie Algebra
}

Takaesu, Toshimitsu

Faculty of Mathematics, Kyushu University

http://hdl. hand le. net/2324/17859

出版情報 : MI Preprint Series. 24，2010-07-06. 九州大学大学院数理学研究院 バージョン：

権利関係: 


\section{Preprint Series}

Kyushu University

The Global COE Program

Math-for-Industry Education \& Research Hub

\section{A Hardy's Uncertainty Principle Lemma in Weak Commutation Relations of Heisenberg-Lie Algebra}

\section{Toshimitsu Takaesu}

MI 2010-24

( Received July 6, 2010 )

Faculty of Mathematics

Kyushu University

Fukuoka, JAPAN 


\title{
A Hardy's Uncertainty Principle Lemma in Weak Commutation Relations of Heisenberg-Lie Algebra
}

\author{
Toshimitsu TAKAESU
}

\author{
Faculty of Mathematics, Kyushu University, \\ Fukuoka, 812-8581, Japan
}

\begin{abstract}
In this article we consider linear operators satisfying a generalized commutation relation of a type of the Heisenberg-Lie algebra. It is proven that a generalized inequality of the Hardy's uncertainty principle lemma follows. Its applications to time operators and abstract Dirac operators are also investigated.
\end{abstract}

Key words : weak commutation relations, Heisenberg-Lie algebra, time operators, Hamiltonians, time-energy uncertainty relation, Dirac operators, essential self-adjointness.

MSC 2010 : 81Q10, 47B25, 46L60.

\section{Introduction and Results}

In this article we investigate a norm-inequality of the linear operators which obey a generalized weak commutation relation of a type of the Heisenberg-Lie algebra, and consider its application to the theory of the time operator [7, 2], and an abstract Dirac operator. Let $\mathbf{X}=\left\{X_{j}\right\}_{j=1}^{N}, \mathbf{Y}=\left\{Y_{j}\right\}_{j=1}^{N}$ and $\mathbf{Z}=\left\{Z_{j}\right\}_{j=1}^{N}$ be symmetric operators on a Hilbert space $\mathcal{H}$. The weak commutator of operators $A$ and $B$ is defined for $\psi \in \mathcal{D}(A) \cap \mathcal{D}(B)$ and $\phi \in \mathcal{D}\left(A^{*}\right) \cap \mathcal{D}\left(B^{*}\right)$ by

$$
[A, B]^{\mathrm{w}}(\phi, \psi)=\left(A^{*} \phi, B \psi\right)-\left(B^{*} \phi, A \psi\right) .
$$

Here the inner product has a linearity of $(\eta, \alpha \psi+\beta \phi)=\alpha(\eta, \psi)+\beta(\eta, \phi)$ for $\alpha, \beta \in \mathbf{C}$. We assume that $(\mathbf{X}, \mathbf{Y}, \mathbf{Z})$ satisfies the following conditions.

(A.1) $Z_{j}, 1 \leq j \leq N$, is bounded operator.

(A.2) Let $\mathcal{D}_{\mathbf{X}}=\cap_{j=1}^{N} \mathcal{D}\left(X_{j}\right)$ and $\mathcal{D}_{\mathbf{Y}}=\cap_{j=1}^{N} \mathcal{D}\left(Y_{j}\right)$. It follows that for $\phi, \psi \in \mathcal{D}_{\mathbf{X}} \cap \mathcal{D}_{\mathbf{Y}}$,

$$
\begin{aligned}
& {\left[X_{j}, Y_{l}\right]^{\mathrm{w}}(\phi, \psi)=\delta_{j, l}\left(\phi, i Z_{j} \psi\right),} \\
& {\left[X_{j}, Z_{l}\right]^{\mathrm{w}}(\phi, \psi)=\left[Y_{j}, Z_{l}\right]^{\mathrm{w}}(\phi, \psi)=0} \\
& {\left[X_{j}, X_{l}\right]^{\mathrm{w}}(\phi, \psi)=\left[Y_{j}, Y_{l}\right]^{\mathrm{w}}(\phi, \psi)=\left[Z_{j}, Z_{l}\right]^{\mathrm{w}}(\phi, \psi)=0 .}
\end{aligned}
$$

Note that $\left[Z_{j}, Z_{l}\right] \psi=0$ follows for $\psi \in \mathcal{H}$, since $Z_{j}, j=1, \cdots, N$, is bounded. In this article we consider an generalization of the inequality

$$
\int_{\mathbf{R}^{N}} \frac{1}{|\mathbf{r}|^{2}}|u(\mathbf{r})|^{2} d \mathbf{r} \leq \frac{4}{(N-2)^{2}} \int_{\mathbf{R}^{N}}|\nabla u(\mathbf{r})|^{2} d \mathbf{r}, \quad N \geq 3 .
$$


This inequality is a basic one of Hardy's uncertainty principle inequalities. For Hardy's uncertainty inequalities, refer to e.g. $[5,6,13]$.

Let us introduce the additional conditions.

(A.3) $X_{j}$ is self-adjoint for all $1 \leq j \leq N$.

(A.4) $X_{i}$ and $Z_{l}$ strongly commutes for all $1 \leq j \leq N$ and $1 \leq l \leq N$.

Sicne $Z_{j}, j=1, \cdots, N$, is bounded self-adjoint operator, we can set $\lambda_{\min }(\mathbf{Z})$ and $\lambda_{\max }(\mathbf{Z})$ by

$$
\begin{aligned}
& \lambda_{\min }(\mathbf{Z})=\min _{1 \leq j \leq N} \inf \sigma\left(Z_{j}\right), \\
& \lambda_{\max }(\mathbf{Z})=\max _{1 \leq j \leq N} \sup \sigma\left(Z_{j}\right),
\end{aligned}
$$

where $\sigma(O)$ denotes the spectrum of the operator $O$.

Theorem 1 Assume (A.1)-(A.4). Let $\Psi \in \mathcal{D}\left(|\mathbf{X}|^{-1}\right) \cap \mathcal{D}_{\mathbf{X}} \cap \mathcal{D}_{\mathbf{Y}}$. Then the following (1) and (2) hold (1) If $N \lambda_{\min }(\mathbf{Z})-2 \lambda_{\max }(\mathbf{Z})>0$, it follows that

$$
\left\||\mathbf{X}|^{-1} \Psi\right\|^{2} \leq \frac{4}{\left(N \lambda_{\min }(\mathbf{Z})-2 \lambda_{\max }(\mathbf{Z})\right)^{2}} \sum_{j=1}^{N}\left\|Y_{j} \Psi\right\|^{2} .
$$

(2) If $2 \lambda_{\min }(\mathbf{Z})-N \lambda_{\max }(\mathbf{Z})>0$, it follows that

$$
\left\||\mathbf{X}|^{-1} \Psi\right\| \leq \frac{4}{\left(2 \lambda_{\min }(\mathbf{Z})-N \lambda_{\max }(\mathbf{Z})\right)^{2}} \sum_{j=1}^{N}\left\|Y_{j} \Psi\right\|^{2} .
$$

Before proving Theorem 1, let us consider the replacement of $\mathbf{X}$ and $\mathbf{Y}$ in Theorem 1. Let us introduce the following conditions substitute for (A.3) and (A.4).

(A.5) $Y_{j}$ is self-adjoint for all $1 \leq j \leq N$.

(A.6) $Y_{i}$ and $Z_{l}$ strongly commutes for all $1 \leq j \leq N$ and $1 \leq l \leq N$.

It is seen from (A.2), that

$$
\left[Y_{j}, X_{l}\right]^{\mathrm{w}}(\phi, \psi)=\delta_{j, l}\left(\phi, i\left(-Z_{j}\right) \psi\right), \quad \phi, \psi \in \mathcal{D}_{\mathbf{X}} \cap \mathcal{D}_{\mathbf{Y}}
$$

Note that $\inf \sigma\left(-Z_{j}\right)=-\sup \left(Z_{j}\right)$ and $\sup \left(-Z_{j}\right)=-\inf \sigma\left(Z_{j}\right)$ follow. Then we obtain a following corollary : 
Corollary 2 Assume (A.1)-(A.2) and (A.5)-(A.6). Let $\Psi \in \mathcal{D}\left(|\mathbf{Y}|^{-1}\right) \cap \mathcal{D}_{\mathbf{X}} \cap \mathcal{D}_{\mathbf{Y}}$. Then the following (1) and (2) hold.

(1) If $2 \lambda_{\min }(\mathbf{Z})-N \lambda_{\max }(\mathbf{Z})>0$, it follows that

$$
\left\||\mathbf{Y}|^{-1} \Psi\right\| \leq \frac{4}{\left(2 \lambda_{\min }(\mathbf{Z})-N \lambda_{\max }(\mathbf{Z})\right)^{2}} \sum_{j=1}^{N}\left\|X_{j} \Psi\right\|^{2}
$$

(2) If $N \lambda_{\min }(\mathbf{Z})-2 \lambda_{\max }(\mathbf{Z})>0$, it follows that

$$
\left\||\mathbf{Y}|^{-1} \Psi\right\| \leq \frac{4}{\left(N \lambda_{\min }(\mathbf{Z})-2 \lambda_{\max }(\mathbf{Z})\right)^{2}} \sum_{j=1}^{N}\left\|X_{j} \Psi\right\|^{2} .
$$

\section{(Proof of Theorem 1)}

(1)Let $\Psi \in \mathcal{D}\left(|\mathbf{X}|^{-1}\right) \cap \mathcal{D}_{\mathbf{X}} \cap \mathcal{D}_{\mathbf{Y}}$. For $\varepsilon>0$ and $t>0$, it is seen that

$$
\left\|\left(Y_{j}-i t X_{j}\left(\mathbf{X}^{2}+\varepsilon\right)^{-1}\right) \Psi\right\|^{2}=\left\|Y_{j} \Psi\right\|^{2}-i t\left[Y_{j}, X_{j}\left(\mathbf{X}^{2}+\varepsilon\right)^{-1}\right]^{\mathrm{w}}(\Psi, \Psi)+t^{2}\left\|X_{j}\left(\mathbf{X}^{2}+\varepsilon\right)^{-1} \Psi\right\|^{2} .
$$

We see that

$$
\left[Y_{j}, X_{j}\left(\mathbf{X}^{2}+\varepsilon\right)^{-1}\right]^{\mathrm{w}}(\Psi, \Psi)=\left[Y_{j}, X_{j}\right]^{\mathrm{w}}\left(\Psi,\left(\mathbf{X}^{2}+\varepsilon\right)^{-1} \Psi\right)+\left[Y_{j},\left(\mathbf{X}^{2}+\varepsilon\right)^{-1}\right]^{\mathrm{w}}\left(X_{j} \Psi, \Psi\right) .
$$

From (A.2) and (A.4), we obtain that

$$
\left[Y_{j}, X_{j}\right]^{\mathrm{w}}\left(\Psi,\left(\mathbf{X}^{2}+\varepsilon\right)^{-1} \Psi\right)=-i\left(\left(\mathbf{X}^{2}+\varepsilon\right)^{-1 / 2} \Psi, Z_{j}\left(\mathbf{X}^{2}+\varepsilon\right)^{-1 / 2} \Psi\right) .
$$

Note that for a symmetric operator $A$ and the non-negative symmetric operator $B$, the resolvent formula $\left[A,(B+\lambda)^{-1}\right]^{\mathrm{w}}(v, u)=[B, A]^{\mathrm{w}}\left((B+\lambda)^{-1} v,(B+\lambda)^{-1} u\right)$ for $\lambda>0$ follows. Then by using this formura, (A.2) and (A.4) yield that

$$
\left[Y_{j},\left(\mathbf{X}^{2}+\varepsilon\right)^{-1}\right]^{\mathrm{w}}\left(X_{j} \Psi, \Psi\right)=2 i\left(X_{j}\left(\mathbf{X}^{2}+\varepsilon\right)^{-1} u, Z_{j} X_{j}\left(\mathbf{X}^{2}+\varepsilon\right)^{-1} u\right)
$$

Since $\left\|\left(Y_{j}-i t X_{j}\left(\mathbf{X}^{2}+\varepsilon\right)^{-1}\right) u\right\|^{2} \geq 0$ and $t>0$, we see from (7), (8) and (9) that

$$
\begin{aligned}
& \left\|Y_{j} \Psi\right\|^{2} \\
& \geq-t^{2}\left\|X_{j}\left(\mathbf{X}^{2}+\varepsilon\right)^{-1} u\right\|^{2}+t\left(\left(\mathbf{X}^{2}+\varepsilon\right)^{-1 / 2} \Psi, Z_{j}\left(\mathbf{X}^{2}+\varepsilon\right)^{-1 / 2} u\right)-2 t\left(X_{j}\left(\mathbf{X}^{2}+\varepsilon\right)^{-1} u, Z_{j} X_{j}\left(\mathbf{X}^{2}+\varepsilon\right)^{-1} \Psi\right) \\
& \geq\left(-t^{2}-2 t \lambda_{\max }(\mathbf{Z})\right)\left\|X_{j}\left(\mathbf{X}^{2}+\varepsilon\right)^{-1} u\right\|^{2}+t \lambda_{\min }(\mathbf{Z})\left\|\left(\mathbf{X}^{2}+\varepsilon\right)^{-1 / 2} \Psi\right\| .
\end{aligned}
$$

Then we have that

$$
\sum_{j=1}^{N}\left\|Y_{j} \Psi\right\|^{2} \geq\left(-t^{2}-2 t \lambda_{\max }(\mathbf{Z})\right)\left\||\mathbf{X}|\left(\mathbf{X}^{2}+\varepsilon\right)^{-1} \Psi\right\|^{2}+t N \lambda_{\min }(\mathbf{Z})\left\|\left(\mathbf{X}^{2}+\varepsilon\right)^{-1 / 2} \Psi\right\|
$$

Note that $\lim _{\varepsilon \rightarrow 0}\left\||\mathbf{X}|\left(\mathbf{X}^{2}+\varepsilon\right)^{-1} \Psi\right\|^{2}=\left\||\mathbf{X}|^{-1} \Psi\right\|$ and $\lim _{\varepsilon \rightarrow 0}\left\|\left(\mathbf{X}^{2}+\varepsilon\right)^{-1 / 2} \Psi\right\|=\left\||\mathbf{X}|^{-1} \Psi\right\|=0$ follow from the spectral decomposition theorem. Then we have

$$
\sum_{j=1}^{N}\left\|Y_{j} \Psi\right\|^{2} \geq\left(-t^{2}+\left(N \lambda_{\min }(\mathbf{Z})-2 \lambda_{\max }(\mathbf{Z})\right) t\right)\left\||\mathbf{X}|^{-1} \Psi\right\| .
$$


By taking $t=\frac{N \lambda_{\min }(\mathbf{Z})-2 \lambda_{\max }(\mathbf{Z})}{2}>0$ in the right side of (12), we obtain (1).

(2) By computing $\left\|\left(Y_{j}+i t X_{j}\left(\mathbf{X}^{2}+\varepsilon\right)^{-1}\right) \Psi\right\|^{2}$ for $t>0$ and $\varepsilon>0$, in a similar way of (1), we see that

$$
\begin{aligned}
& \left\|Y_{j} \Psi\right\|^{2} \\
& \geq-t^{2}\left\|X_{j}\left(\mathbf{X}^{2}+\varepsilon\right)^{-1} u\right\|^{2}-t\left(\left(\mathbf{X}^{2}+\varepsilon\right)^{-1 / 2} \Psi, Z_{j}\left(\mathbf{X}^{2}+\varepsilon\right)^{-1 / 2} u\right)+2 t\left(X_{j}\left(\mathbf{X}^{2}+\varepsilon\right)^{-1} u, Z_{j} X_{j}\left(\mathbf{X}^{2}+\varepsilon\right)^{-1} \Psi\right) \\
& \geq\left(-t^{2}+2 t \lambda_{\min }(\mathbf{Z})\right)\left\|X_{j}\left(\mathbf{X}^{2}+\varepsilon\right)^{-1} u\right\|^{2}-t \lambda_{\max }(\mathbf{Z})\left\|\left(\mathbf{X}^{2}+\varepsilon\right)^{-1 / 2} \Psi\right\|
\end{aligned}
$$

Then by taking $\varepsilon \rightarrow 0$ in the right side of (13), it follows that

$$
\sum_{j=1}^{N}\left\|Y_{j} \Psi\right\|^{2} \geq\left(-t^{2}+\left(2 \lambda_{\min }(\mathbf{Z})-N \lambda_{\max }(\mathbf{Z})\right) t\right)\left\||\mathbf{X}|^{-1} \Psi\right\| .
$$

By taking $t=\frac{\left(2 \lambda_{\min }(\mathbf{Z})-N \lambda_{\max }(\mathbf{Z})\right)}{2}>0$ in (14), we obtain (2).

\section{Applications}

\subsection{Time-Energy Uncertainty inequality}

In this subsection we consider an applicaion to the theory of time operators [2, 7]. Let $H, T$, and $C$ be linear operators on a Hilbert space $H$. It is said that $H$ has the weak time operator $T$ with the uncommutative factor $C$ if $(H, T, C)$ satisfy the following conditions.

(T.1) $H$ and $T$ are symmetric.

(T.2) $\mathrm{C}$ is bounded and self-adjoint.

(T.3) It follows that for $\phi, \psi \in \mathcal{D}(H) \cap \mathcal{D}(T)$,

$$
[T, H]^{\mathrm{w}}(\phi, \psi)=(\phi, C \psi)
$$

(T.4)

$$
\delta_{C}:=\inf _{\psi \in(\operatorname{ker} C)^{\perp} \backslash\{0\}} \frac{|(\Psi, C \Psi)|}{\|\psi\|^{2}}>0 .
$$

Assume that $(H, T, C)$ satisfies (T.1)-(T.4). Then by using $\|A u\|\|B u\| \geq|\operatorname{Im}(A u, B u)| \geq \frac{1}{2}\left|[A, B]^{\mathrm{w}}(u, u)\right|$, it is seen that $(\mathrm{H}, \mathrm{T}, \mathrm{C})$ satisfies the time-energy uncertainty inequality ([2], Proposition4.1):

$$
\frac{\left\|\left(H-<H>_{\psi}\right) \psi\right\|\left\|\left(T-<T>_{\psi}\right) \psi\right\|}{\|\psi\|^{2}} \geq \frac{\delta_{C}}{2}, \quad \psi \in \mathcal{D}(H) \cap \mathcal{D}(T),
$$

where $\left\langle O>_{\psi}=(\psi, O \psi)\right.$. From (2) in Theorem 1 and (1) in Corollary 2, we obtain another type of the inequality between $T$ and $H$ : 


\section{Corollary 3 (Time-Energy Uncertainty Inequalities)}

Assume (T.1)-(T.3). Then the following (i) and (ii) hold.

(i) If $T$ is self-adjoint, $C$ and $T$ strongly commute, and $\sup \sigma(C)<2 \inf \sigma(C)$, it follows that for $\psi \in$ $\mathcal{D}\left(|T|^{-1}\right) \cap \mathcal{D}(T) \cap \mathcal{D}(H)$,

$$
\left\||T|^{-1} \psi\right\| \leq \frac{2}{2 \inf \sigma(C)-\sup \sigma(C)}\|H \Psi\| .
$$

(ii) If $H$ is self-adjoint, $C$ and $H$ strongly commute, and $\sup \sigma(C)<2 \inf \sigma(C)$, it follows that for $\psi \in$ $\mathcal{D}\left(|H|^{-1}\right) \cap \mathcal{D}(H) \cap \mathcal{D}(T)$,

$$
\left\||H|^{-1} \psi\right\| \leq \frac{2}{2 \inf \sigma(C)-\sup \sigma(C)}\|T \Psi\| .
$$

\subsection{Abstract Dirac Operators with Coulomb Potential}

Next tlt us consider the application to abstract Dirac operators. We consider the self-adjoint operators $\mathbf{P}=\left\{P_{j}\right\}_{j=1}^{N}$ and $\mathbf{Q}=\left\{Q_{j}\right\}_{j=1}^{N}$ on a Hilbert space $\mathcal{H}$. Let us set a subspace $\mathcal{D} \subset \cap_{j, l}\left(\mathcal{D}\left(P_{j}\right) \cap \mathcal{D}\left(Q_{l}\right)\right)$. It is said that $(\mathcal{H}, \mathcal{D}, \mathbf{P}, \mathbf{Q})_{N}$ is the weak representaion of the CCR with degree $N$, if $\mathcal{D}$ is dense in $\mathcal{H}$ and it follows that for $\phi, \psi \in \mathcal{D}$,

$$
\begin{aligned}
& {\left[P_{j}, Q_{l}\right]^{\mathrm{w}}(\phi, \psi)=i \delta_{j, l}(\phi, \psi),} \\
& {\left[P_{j}, P_{l}\right]^{\mathrm{w}}(\phi, \psi)=\left[Q_{j}, Q_{l}\right]^{\mathrm{w}}(\phi, \psi)=0 .}
\end{aligned}
$$

Let us define an abstract Dirac operator as follows. Let $(\mathcal{H}, \mathcal{D}, \mathbf{P}, \mathbf{Q})_{3}$ be the weak representation of the CCR with degree three. Let $\mathbf{A}=\left\{A_{j}\right\}_{j=1}^{3}$ and $B$ be the bounded self-adjoint operators on a Hilbert space $\mathcal{K}$. Here $\mathbf{A}=\left\{A_{j}\right\}_{j=1}^{3}$ and $B$ satisfy the canonical anti-commutation relations $\left\{A_{j}, A_{l}\right\}=2 \delta_{j, l}$, $\left\{A_{j}, B\right\}=0, B^{2}=I_{\mathcal{K}}$ where $I_{\mathcal{K}}$ is the identity operator on $\mathcal{K}$. The state Hilbert space space is defined by $\mathcal{H}_{\text {Dirac }}=\mathcal{K} \otimes \mathcal{H}$. The free abstract Dirac operator is defiend by

$$
H_{0}=\sum_{j=1}^{3} A_{j} \otimes P_{j}+B \otimes M .
$$

Here we assume the following condition.

(D.1) $P_{j}$ and $P_{l}$ strongly commute for $1 \leq j \leq 3,1 \leq l \leq 3 . P_{j}, 1 \leq j \leq 3$, and $M$ strongly commute.

Then it is seen that $H_{0}^{2} \Psi=\left(\mathbf{P}^{2}+M^{2}\right) \Psi$ for $\Psi \in \mathcal{D}$. The abstract Dirac Operator with the Coulomb potential is defined by

$$
H(\kappa)=H_{0}+\kappa I_{\mathcal{K}} \otimes|\mathbf{Q}|^{-1},
$$

where $\kappa \in \mathbf{R}$ is a parameter called the coupling constant. We assume that the following condition

(D.2) It follows that $\mathcal{D} \subset \mathcal{D}\left(\left.\mathbf{Q}\right|^{-1}\right)$.

Then it follows from (1) in Theorem 1 that for $\psi \in \mathcal{D}$,

$$
\left\|I_{\mathcal{K}} \otimes|\mathbf{Q}|^{-1} \psi\right\|^{2} \leq 4 \sum_{j=1}^{3}\left\|P_{j} \Psi\right\|^{2} \leq 4\left\|H_{0} \Psi\right\|^{2} .
$$

Hence by the Kato-Rellich theorem, we obtaine the following corollary. 
Corollary 4 Assume (D.1) and (D.2). Then for $|\kappa|<\frac{1}{2}, H(\kappa)$ is essentially self-adjoint on $\mathcal{D}$.

\section{Acknowledgments}

It is pleasure to thank assistant professor Akito Suzuki and associate professor Fumio Hiroshima for their advice and comments.

\section{References}

[1] A.Arai, Mathematical principles of quantum phenamena, Asakura-syoten, 2005. (in japanese)

[2] A.Arai, Generalized weak Weyl relation and decay of quantum dynamics, Rev. Math. Phys. 17 (2005) 10711109.

[3] A.Arai, Heisenberg Operators, invariant domains and Heisenberg equations of motion, Rev. Math. Phys 19 (2007) 1045-1069.

[4] A.Arai, Spectrum of time operators. Lett. Math. Phys. 80 211-221 (2007).

[5] G.B.Folland and A.Sitaram, The uncertainty principle : A mathematical survey, J. Fourier Anal. Appl. 3 (1997) 207-238.

[6] V.Havin and B.Joricke, The uncertainty principle in harmonic analysis, Springer 1994.

[7] M.Miyamoto, A generalized Weyl relation approach to the time operator and its connection to the survival probability, J. Math. Phys 42 (2001) 1038-1052.

[8] J.G.Muga, R.S.Mayato,and I.L.Egsquiza (eds.), Time in quantum mechanics. Springer 2002.

[9] P.Pfeifer and J. Frölich, Generalized time-energy uncertainty relations and bounds on lifetimes of resonances, Rev. Mod. Phys. 67 (1995) 759-779.

[10] M.Reed and B.Simon, Methods of Modern Mathematical Physics Vol.II, Academic Press, 1979.

[11] K.Schmüdgen, On the Heisenberg commutationn relationhn. I, J. Funct. Anal. 50 (1983) 8-49.

[12] B. Thaller, The Dirac equation, Springer, 1992.

[13] S.Thangavelu, An introduction to the uncertainty principle : Hardy's theorem on Lie groups, Birkhäuser, 2004. 


\section{List of MI Preprint Series, Kyushu University}

The Global COE Program

Math-for-Industry Education \& Research Hub

MI

MI2008-1 Takahiro ITO, Shuichi INOKUCHI \& Yoshihiro MIZOGUCHI

Abstract collision systems simulated by cellular automata

MI2008-2 Eiji ONODERA

The intial value problem for a third-order dispersive flow into compact almost

Hermitian manifolds

MI2008-3 Hiroaki KIDO

On isosceles sets in the 4-dimensional Euclidean space

MI2008-4 Hirofumi NOTSU

Numerical computations of cavity flow problems by a pressure stabilized characteristiccurve finite element scheme

MI2008-5 Yoshiyasu OZEKI

Torsion points of abelian varieties with values in nfinite extensions over a padic field

MI2008-6 Yoshiyuki TOMIYAMA

Lifting Galois representations over arbitrary number fields

MI2008-7 Takehiro HIROTSU \& Setsuo TANIGUCHI

The random walk model revisited

MI2008-8 Silvia GANDY, Masaaki KANNO, Hirokazu ANAI \& Kazuhiro YOKOYAMA Optimizing a particular real root of a polynomial by a special cylindrical algebraic decomposition

MI2008-9 Kazufumi KIMOTO, Sho MATSUMOTO \& Masato WAKAYAMA

Alpha-determinant cyclic modules and Jacobi polynomials 
MI2008-10 Sangyeol LEE \& Hiroki MASUDA

Jarque-Bera Normality Test for the Driving Lévy Process of a Discretely Observed Univariate SDE

MI2008-11 Hiroyuki CHIHARA \& Eiji ONODERA

A third order dispersive flow for closed curves into almost Hermitian manifolds

MI2008-12 Takehiko KINOSHITA, Kouji HASHIMOTO and Mitsuhiro T. NAKAO

On the $L^{2}$ a priori error estimates to the finite element solution of elliptic problems with singular adjoint operator

MI2008-13 Jacques FARAUT and Masato WAKAYAMA

Hermitian symmetric spaces of tube type and multivariate Meixner-Pollaczek polynomials

MI2008-14 Takashi NAKAMURA

Riemann zeta-values, Euler polynomials and the best constant of Sobolev inequality

MI2008-15 Takashi NAKAMURA

Some topics related to Hurwitz-Lerch zeta functions

MI2009-1 Yasuhide FUKUMOTO

Global time evolution of viscous vortex rings

MI2009-2 Hidetoshi MATSUI \& Sadanori KONISHI

Regularized functional regression modeling for functional response and predictors

MI2009-3 Hidetoshi MATSUI \& Sadanori KONISHI

Variable selection for functional regression model via the $L_{1}$ regularization

MI2009-4 Shuichi KAWANO \& Sadanori KONISHI

Nonlinear logistic discrimination via regularized Gaussian basis expansions

MI2009-5 Toshiro HIRANOUCHI \& Yuichiro TAGUCHII

Flat modules and Groebner bases over truncated discrete valuation rings 
MI2009-6 Kenji KAJIWARA \& Yasuhiro OHTA

Bilinearization and Casorati determinant solutions to non-autonomous $1+1$ dimensional discrete soliton equations

\section{MI2009-7 Yoshiyuki KAGEI}

Asymptotic behavior of solutions of the compressible Navier-Stokes equation around the plane Couette flow

MI2009-8 Shohei TATEISHI, Hidetoshi MATSUI \& Sadanori KONISHI

Nonlinear regression modeling via the lasso-type regularization

MI2009-9 Takeshi TAKAISHI \& Masato KIMURA

Phase field model for mode III crack growth in two dimensional elasticity

MI2009-10 Shingo SAITO

Generalisation of Mack's formula for claims reserving with arbitrary exponents for the variance assumption

MI2009-11 Kenji KAJIWARA, Masanobu KANEKO, Atsushi NOBE \& Teruhisa TSUDA Ultradiscretization of a solvable two-dimensional chaotic map associated with the Hesse cubic curve

\section{MI2009-12 Tetsu MASUDA}

Hypergeometric T -functions of the q-Painlevé system of type $E_{8}^{(1)}$

MI2009-13 Hidenao IWANE, Hitoshi YANAMI, Hirokazu ANAI \& Kazuhiro YOKOYAMA A Practical Implementation of a Symbolic-Numeric Cylindrical Algebraic Decomposition for Quantifier Elimination

MI2009-14 Yasunori MAEKAWA

On Gaussian decay estimates of solutions to some linear elliptic equations and its applications

MI2009-15 Yuya ISHIHARA \& Yoshiyuki KAGEI

Large time behavior of the semigroup on $L^{p}$ spaces associated with the linearized compressible Navier-Stokes equation in a cylindrical domain 
MI2009-16 Chikashi ARITA, Atsuo KUNIBA, Kazumitsu SAKAI \& Tsuyoshi SAWABE Spectrum in multi-species asymmetric simple exclusion process on a ring

MI2009-17 Masato WAKAYAMA \& Keitaro YAMAMOTO

Non-linear algebraic differential equations satisfied by certain family of elliptic functions

MI2009-18 Me Me NAING \& Yasuhide FUKUMOTO

Local Instability of an Elliptical Flow Subjected to a Coriolis Force

MI2009-19 Mitsunori KAYANO \& Sadanori KONISHI

Sparse functional principal component analysis via regularized basis expansions and its application

MI2009-20 Shuichi KAWANO \& Sadanori KONISHI

Semi-supervised logistic discrimination via regularized Gaussian basis expansions

MI2009-21 Hiroshi YOSHIDA, Yoshihiro MIWA \& Masanobu KANEKO

Elliptic curves and Fibonacci numbers arising from Lindenmayer system with symbolic computations

MI2009-22 Eiji ONODERA

A remark on the global existence of a third order dispersive flow into locally Hermitian symmetric spaces

MI2009-23 Stjepan LUGOMER \& Yasuhide FUKUMOTO

Generation of ribbons, helicoids and complex scherk surface in laser-matter Interactions

MI2009-24 Yu KAWAKAMI

Recent progress in value distribution of the hyperbolic Gauss map

MI2009-25 Takehiko KINOSHITA \& Mitsuhiro T. NAKAO

On very accurate enclosure of the optimal constant in the a priori error estimates for $H_{0}^{2}$-projection 
MI2009-26 Manabu YOSHIDA

Ramification of local fields and Fontaine's property (Pm)

MI2009-27 Yu KAWAKAMI

Value distribution of the hyperbolic Gauss maps for flat fronts in hyperbolic three-space

MI2009-28 Masahisa TABATA

Numerical simulation of fluid movement in an hourglass by an energy-stable finite element scheme

MI2009-29 Yoshiyuki KAGEI \& Yasunori MAEKAWA Asymptotic behaviors of solutions to evolution equations in the presence of translation and scaling invariance

MI2009-30 Yoshiyuki KAGEI \& Yasunori MAEKAWA

On asymptotic behaviors of solutions to parabolic systems modelling chemotaxis

MI2009-31 Masato WAKAYAMA \& Yoshinori YAMASAKI

Hecke's zeros and higher depth determinants

MI2009-32 Olivier PIRONNEAU \& Masahisa TABATA

Stability and convergence of a Galerkin-characteristics finite element scheme of lumped mass type

MI2009-33 Chikashi ARITA

Queueing process with excluded-volume effect

MI2009-34 Kenji KAJIWARA, Nobutaka NAKAZONO \& Teruhisa TSUDA

Projective reduction of the discrete Painlevé system of type $\left(A_{2}+A_{1}\right)^{(1)}$

MI2009-35 Yosuke MIZUYAMA, Takamasa SHINDE, Masahisa TABATA \& Daisuke TAGAMI Finite element computation for scattering problems of micro-hologram using DtN map 
MI2009-36 Reiichiro KAWAI \& Hiroki MASUDA

Exact simulation of finite variation tempered stable Ornstein-Uhlenbeck processes

MI2009-37 Hiroki MASUDA

On statistical aspects in calibrating a geometric skewed stable asset price model

MI2010-1 Hiroki MASUDA

Approximate self-weighted LAD estimation of discretely observed ergodic OrnsteinUhlenbeck processes

MI2010-2 Reiichiro KAWAI \& Hiroki MASUDA

Infinite variation tempered stable Ornstein-Uhlenbeck processes with discrete observations

MI2010-3 Kei HIROSE, Shuichi KAWANO, Daisuke MIIKE \& Sadanori KONISHI Hyper-parameter selection in Bayesian structural equation models

MI2010-4 Nobuyuki IKEDA \& Setsuo TANIGUCHI

The Itô-Nisio theorem, quadratic Wiener functionals, and 1-solitons

MI2010-5 Shohei TATEISHI \& Sadanori KONISHI

Nonlinear regression modeling and detecting change point via the relevance vector machine

MI2010-6 Shuichi KAWANO, Toshihiro MISUMI \& Sadanori KONISHI

Semi-supervised logistic discrimination via graph-based regularization

MI2010-7 Teruhisa TSUDA

UC hierarchy and monodromy preserving deformation

MI2010-8 Takahiro ITO

Abstract collision systems on groups

MI2010-9 Hiroshi YOSHIDA, Kinji KIMURA, Naoki YOSHIDA, Junko TANAKA \& Yoshihiro MIWA

An algebraic approach to underdetermined experiments 
MI2010-10 Kei HIROSE \& Sadanori KONISHI

Variable selection via the grouped weighted lasso for factor analysis models

MI2010-11 Katsusuke NABESHIMA \& Hiroshi YOSHIDA

Derivation of specific conditions with Comprehensive Groebner Systems

MI2010-12 Yoshiyuki KAGEI, Yu NAGAFUCHI \& Takeshi SUDOU

Decay estimates on solutions of the linearized compressible Navier-Stokes equation around a Poiseuille type flow

MI2010-13 Reiichiro KAWAI \& Hiroki MASUDA

On simulation of tempered stable random variates

MI2010-14 Yoshiyasu OZEKI

Non-existence of certain Galois representations with a uniform tame inertia weight

MI2010-15 Me Me NAING \& Yasuhide FUKUMOTO

Local Instability of a Rotating Flow Driven by Precession of Arbitrary Frequency

MI2010-16 Yu KAWAKAMI \& Daisuke NAKAJO

The value distribution of the Gauss map of improper affine spheres

MI2010-17 Kazunori YASUTAKE

On the classification of rank 2 almost Fano bundles on projective space

MI2010-18 Toshimitsu TAKAESU

Scaling limits for the system of semi-relativistic particles coupled to a scalar bose field

MI2010-19 Reiichiro KAWAI \& Hiroki MASUDA

Local asymptotic normality for normal inverse Gaussian Lévy processes with high-frequency sampling

MI2010-20 Yasuhide FUKUMOTO, Makoto HIROTA \& Youichi MIE

Lagrangian approach to weakly nonlinear stability of an elliptical flow 
MI2010-21 Hiroki MASUDA

Approximate quadratic estimating function for discretely observed Lévy driven SDEs with application to a noise normality test

MI2010-22 Toshimitsu TAKAESU

A Generalized Scaling Limit and its Application to the Semi-Relativistic Particles System Coupled to a Bose Field with Removing Ultraviolet Cutoffs

MI2010-23 Takahiro ITO, Mitsuhiko FUJIO, Shuichi INOKUCHI \& Yoshihiro MIZOGUCHI Composition, union and division of cellular automata on groups

MI2010-24 Toshimitsu TAKAESU

A Hardy's Uncertainty Principle Lemma in Weak Commutation Relations of Heisenberg-Lie Algebra 